\title{
The Second Virial Coefficient for the Realistic Pair Potential*
}

\author{
Harold W. Woolley \\ Institute for Basic Standards, National Bureau of Standards, Washington, D.C. 20234
}

(April 8, 1969)

\begin{abstract}
An analytical formulation for the second virial coefficient is given for a spherically symmetric potential function of a simple form which has been called a realistic pair potential. This potential, given as an inverse relationship with $r$ as a function of $U$, is capable of being much softer than a LennardJones in the extreme of close approach. Differing forms of the result are given, including an expression by means of the generalized hypergeometric series. The result is also expressed in terms of the second virial coefficient for the $(12,6)$ potential.
\end{abstract}

Key words:' Hypergeometric series; London interaction; pair potential function; realistic potential; second virial coefficient; spherical potential.

One of the uses of a pair potential function is for the representation or prediction of the second virial coefficient contribution to the equation of state due to the two molecules having the specified interaction potential energy. Various potential functions have been used with some success, the most notable of which may be the Lennard-Jones (especially the 12,6), the Morse function, and the exp-6.

In spite of the varied nature of the different functions, it is sometimes pointed out that there is so much similarity in second virial behavior for them that it is difficult to select one in preference to another on the basis of second virials alone. Even if this be true, there still may be some interest in yet another potential for which the second virial may be formulated explicitly, particularly since this formulation can be made in terms of already tabulated second virial co- efficients for the $(12,6)$ function.

A potential function has been introduced earlier [1] ${ }^{1}$ in which the value of $r / r_{m}$ is given as a function of energy for the usual $1 / r^{6}$ type of London interaction according to the relation

$\left(r / r_{m}\right)^{3}=\phi^{-1 / 2} \exp \left[-3 e_{1}(\phi-1)-3 e_{2}(\phi-1)^{2} ..\right]$.

The quantity $\phi$ is related to the energy $U$ according to the equation

$$
U / \epsilon=(\phi-1)^{2}-1
$$

\footnotetext{
*This research was supported in part by the United States Air Force.

1 Figures in brackets indicate the literature references at the end of this paper.
}

The range of values of $\phi$ is from zero for very large $r$ where $U$ is zero, through $\phi=1$ for $r=r_{m}$ where $U$ has the value $-\epsilon$, and to very large values where $U$ becomes very large as $r$ becomes very small, according to the expected pattern of behavior.

The second viral coefficient may for the present simple case of a spherical potential function be written as

$$
B=N_{0} \int_{0}^{\infty}(1-\exp (-U / k T)) .2 \pi r^{2} d r
$$

if the pair is composed of like molecules. Integration by parts transforms the integral to

$$
B=(2 \pi / 3)\left(N_{0} / k T\right) \int_{0}^{\infty}\left[r^{3}\right] \exp (-U / k T) d U
$$

where the quantity $\left[r^{3}\right]$ is a function of $U$ and represents the simple $r^{3}$ value in the positive energy region but represents the difference of $r^{3}$ for the repulsion side of the potential valley minus $r^{3}$ for the attractive side if the potential energy is negative. This double-valued character is removed if the variable $\phi$ is introduced, since the integral then is

$$
\begin{aligned}
B=(2 \pi / 3) & N_{0}(2 \epsilon / k T) \\
& \int_{0}^{\infty} r^{3} \exp \left[-\left(\phi^{2}-2 \phi\right) \epsilon / k T\right](\phi-1) d \phi
\end{aligned}
$$

with $\phi$ advancing simply from zero to infinity and with $r^{3}$ having its ordinary algebraic meaning.

If we now introduce the present potential in the form 


$$
\left(r / r_{m}\right)^{3}=\phi^{-1 / 2} \exp \left(-a \phi^{2}-b \phi+c\right)
$$

where $a=3 e_{2}, b=3 e_{1}-6 e_{2}$, and $c=3 e_{1}-3 e_{2}$, we have, with $T^{*}$ for $k T / \epsilon$,

$$
\begin{array}{r}
B=(2 \pi / 3) N_{0} r_{m}^{3}\left(2 / T^{*}\right) \int_{0}^{\infty} \exp \left[c-\left(b-2 / T^{*}\right) \phi\right. \\
\left.-\left(a+1 / T^{*}\right) \phi^{2}\right](\phi-1) \phi^{-1 / 2} d \phi
\end{array}
$$

Expansion of the exponential involving the first power of $\phi$ using $\beta=b+2 / T^{*}$ and $\alpha=a+1 / T^{*}$ gives

$$
\begin{aligned}
B=(2 \pi / 3) N_{0} r_{m}^{3}\left(2 / T^{*}\right) & \int_{0}^{\infty} \exp \left[c-\alpha \phi^{2}\right] \\
& \sum_{n=0}^{\infty} \frac{\beta^{n} \phi^{n}}{n !}(\phi-1) \phi^{-1 / 2} d \phi .
\end{aligned}
$$

The substitution of $x$ for $\phi^{2}$ puts the result in the form

$$
\begin{aligned}
B=(2 \pi / 3) N_{0} r_{m}^{3}\left(e^{c} / T^{*}\right) & \sum_{n=0}^{\infty} \frac{\beta^{n}}{n !} \\
& \int_{0}^{\infty} e^{-\alpha x}\left(x^{n / 2-1 / 4}-x^{n / 2-3 / 4}\right) d x
\end{aligned}
$$

or

$$
\begin{aligned}
& B=(2 \pi / 3) N_{0} r_{m}^{3}\left(e^{c} / T^{*}\right) \sum_{n=0}^{\infty} \frac{\beta^{n}}{n !} \\
& {\left[\alpha^{-n / 2-3 / 4} \Gamma(n / 2+3 / 4)-\alpha^{-n / 2-1 / 4} \Gamma(n / 2+1 / 4)\right] .}
\end{aligned}
$$

This can be written as $B=b_{m} .\left(B / b_{m}\right)$ with $b_{m}=(2 \pi / 3)$ $N_{0} r_{m}^{3}$, and

$$
\begin{gathered}
B / b_{m}=\left(e^{c} / T^{*}\right)\left[\alpha^{-3 / 4} \sum_{n=0}^{\infty} \Gamma(n / 2+3 / 4)\left(4 / T^{* *}\right)^{n / 2} / n !\right. \\
\left.-\alpha^{-1 / 4} \sum_{n=0}^{\infty} \Gamma(n / 2+1 / 4)\left(4 / T^{* *}\right)^{n / 2} / n !\right]
\end{gathered}
$$

where $T^{* *}$ is an abbreviation for $4 \alpha / \beta^{2}$.

Another formulation can be given by which the summations may be avoided by various relationships according to which the first summation is equal to

$$
-(\sqrt{2})\left(T^{* *}\right)^{5 / 4}\left[(3 / 8) B^{*}\left(T^{* *}\right)+4 B_{1}^{*}\left(T^{* *}\right)+2 B_{2}^{*}\left(T^{* *}\right)\right]
$$

and the second is

$$
-\frac{\sqrt{2}}{2}\left(T^{* *}\right)^{3 / 4}\left[B^{*}\left(T^{* *}\right)+4 B_{1}^{*}\left(T^{* *}\right)\right],
$$

where $B^{*}, B_{1}^{*}$, and $B_{2}^{*}$ are the usually tabulated second virial coefficients and dimensionless derivatives for the Lennard-Jones $(12,6)$ potential function.

Using these, (11) can be written

$$
\begin{gathered}
\frac{B}{b_{m}}=-\frac{e^{c}}{T^{*}} \alpha^{-1 / 4}\left(T^{* *}\right)^{3 / 4} \sqrt{2}\left\{\left(1 / 2+\frac{3}{8} \alpha^{-1 / 2} T^{* * 1 / 2}\right)\right. \\
B^{*}\left(T^{* *}\right)+\left(2+4 \alpha^{-1 / 2} T^{* * 1 / 2}\right) B_{1}^{*}\left(T^{* *}\right) \\
\left.+2 \alpha^{-1 / 2} T^{* * 1 / 2} B_{2}^{*}\left(T^{* *}\right)\right\}
\end{gathered}
$$

or as

$$
\begin{aligned}
& B / b_{m}=-\frac{e^{c}}{\left[2-b T^{*}\right]^{2}} \sqrt{\frac{1+a T^{*}}{2-b T^{*}}} \\
&\left\{\left[4+(3-2 b) T^{*}\right] B^{*}\left(T^{* *}\right)\right. \\
&\left.+8\left[2+(4-b) T^{*}\right] B_{1}^{*}\left(T^{* *}\right)+16 T^{*} B_{2}^{*}\left(T^{* *}\right)\right\}
\end{aligned}
$$

Another arrangement of the result can be arrived at in the following way. If the second sum in the expression for $B / b_{m}$ is written out in detail through several terms, it is possible to recognize that there is a relation to the generalized hypergeometric function and the confluent hypergeometric function in particular. With $\tau$ representing $\left(4 / T^{* *}\right)^{1 / 2}$, the summation becomes

$\Gamma(1 / 4)\left[F_{1}\left(1 / 4 ; 1 / 2 ; \tau^{2} / 4\right)\right]$

$$
+\Gamma(3 / 4)\left[{ }_{1} F_{1}\left(3 / 4 ; 3 / 2 ; \tau^{2} / 4\right)\right] .
$$

which also has a relationship to Bessel functions [2] We further note that if the ordinary hypergeometric function $F(\alpha, \beta ; \gamma ; \xi)$ with $\xi=\eta / \beta$ is considered and the limit taken as $\beta^{-1} \rightarrow 0$, the result is found to be ${ }_{1} F_{1}(\alpha ; \gamma ; \eta)$. A function equivalent to the original hypergeometric function according to a standard relation is $(1-\xi)^{\gamma-\alpha-\beta} F(\gamma-\alpha, \gamma-\beta ; \gamma ; \xi)$. A similar use of $\xi=\eta / \beta$ and a passing to the limit $\beta^{-1} \rightarrow 0$ produces an equivalent form $e^{\eta_{1} F_{1}}(\gamma-\alpha, \gamma ;-\eta)$. Then for the case with $\alpha=\frac{1}{2} \gamma$ the equating of the two expressions leads to

$$
e^{-\eta / 2}{ }_{1} F_{1}\left(\frac{1}{2} \gamma ; \gamma ; \eta\right)=e^{\eta / 2}{ }_{1} F_{1}^{\prime}\left(\frac{1}{2} \gamma ; \gamma ;-\eta\right)
$$

from which it is evident that both expressions, on either side of the equation, are even functions of $\eta$. It would appear plausible that a series involving even powers only would need half as many terms to attain a given quality of approximation as a related one with both odd and even powers. Thus, we would presume that a more rapidly convergent form for the result should exist and might be looked for. For this purpose we examine the differential equation [2]

$$
\alpha y=(\gamma-x) y^{\prime}+x y^{\prime \prime},
$$

for which $y={ }_{1} F_{1}(\alpha ; \gamma ; x)$ is known to be a solution. For the special case with $\alpha=\frac{1}{2} \gamma$, if we use $z=e^{-x / 2} y$ or $y=e^{x / 2} z$ and substitute into the differential equation, we obtain

$$
x z^{\prime \prime}+\gamma z^{\prime}-\frac{1}{4} x z=0
$$

Solution in series by the method of Frobenius gives 
$z=1+\frac{1}{1 \cdot \delta}\left(\frac{x}{4}\right)^{2}+\frac{1}{1 \cdot 2 \cdot \delta(\delta+1)}\left(\frac{x}{4}\right)^{4}$

$$
+\frac{1}{1 \cdot 2 \cdot 3 \cdot \delta(\delta+1)(\delta+2)}\left(\frac{x}{4}\right)^{6}+\text {. }
$$

or

$$
z={ }_{0} F_{1}\left(\delta ;\left(\frac{x}{4}\right)^{2}\right)
$$

where $\delta=(1+\gamma) / 2$, according to the notation [3] for the generalized hypergeometric series.

In summary, the final series obtained may be substituted into the earlier expressions with the result that the second virial coefficient may be obtained from

$$
B / b_{m}=e^{c} T^{*-1}\left[\alpha^{-3 / 4} Y_{1}(\tau)-\alpha^{-1 / 4} Y_{0}(\tau)\right]
$$

where

$Y_{0}(\tau)=\left[\Gamma(1 / 4)_{0} F_{1}\left(3 / 4 ; \tau^{4} / 256\right)\right.$

$$
\left.+\Gamma(3 / 4) \tau_{0} F_{1}\left(5 / 4 ; \tau^{4} / 256\right)\right] \exp \left(\tau^{2} / 8\right)
$$

with $Y_{1}(\tau)$ obtainable by differentiation according to $Y_{1}(\tau)=d Y_{0}(\tau) / d \tau$, and with $\tau=\left(4 / T^{* *}\right)^{1 / 2}$.

It may be seen that this particular hypergeometric function has a simple rule for differentiation; namely,

$$
d\left[{ }_{0} F_{1}(a ; x)\right] / d x=a^{-1}{ }_{0} F_{1}(a+1 ; x) .
$$

Thus a procedure may be found to evaluate $B / b_{m}$ and any of its derivatives which are of interest.

\section{References}

[1] Woolley, H. W., J. Chem. Phys. 37, 1307 (1962).

[2] Ince, E. L., Ordinary Differential Equations [Longmans, Green and Co., 1926] (Dover Publications, Inc., p. 180, 1956)

[3] Bateman Manuscript Project, Higher Transcendental Functions, Vol. 1, p. 182, A. Erdely, Ed. (McGraw-Hill Book Company, Inc., New York, 1953).

(Paper 73A4-560) 